\title{
Oscillation probability fits using Near Detector data at a Neutrino Factory
}

\author{
Andrew Laing*, F.J.P. Soler ${ }^{\dagger}$ \\ Department of Physics and Astronomy, University of Glasgow, Glasgow, G12 8QQ, U.K. \\ a.laing@physics.gla.ac.uk
}

In this article we present the results of oscillation probability fits using Near Detector data at a Neutrino Factory. The method relies on the extraction of the beam flux at the Near Detector and using these data to predict the number of events observed at a Far Detector as a function of energy. Comparison with the observed events with the Far Detector can be used to extract the neutrino oscillation parameters. Error contour plots in the $\theta_{13}-\delta$ plane shall be extracted from the fits using "true" Monte Carlo data and also assuming realistic detector smearing for values of $\sin ^{2} 2 \theta_{13}$ and the CP phase $\delta$.

10th International Workshop on Neutrino Factories, Super beams and Beta beams June 30 - July 52008

Valencia, Spain

\footnotetext{
* Speaker.

${ }^{\dagger}$ The authors are grateful to the Science and Technologies Facilities Council for financial support.
} 


\section{Introduction}

The $v_{e} \rightarrow v_{\mu}$ (or $\bar{v}_{e} \rightarrow \bar{v}_{\mu}$ ) neutrino oscillation probability in matter at a distance $L$ may be parameterised by [1]: $P_{v_{e} v_{\mu}\left(\bar{v}_{e} \bar{v}_{\mu}\right)}\left(\theta_{13}, \delta\right)=P_{1}+P_{2}+P_{3}+P_{4}$, with

$$
\begin{aligned}
& P_{1}=s_{23}^{2} \sin ^{2} 2 \theta_{13}\left(\frac{\Delta_{13}}{B_{\mp}}\right)^{2} \sin ^{2}\left(\frac{B_{\mp} L}{2}\right) \\
& P_{2}=c_{23}^{2} \sin ^{2} 2 \theta_{12}\left(\frac{\Delta_{12}}{A}\right)^{2} \sin ^{2}\left(\frac{A L}{2}\right) \\
& P_{3}=J \cos \delta \cos \left(\frac{\Delta_{13} L}{2}\right)\left(\frac{\Delta_{12}}{A} \frac{\Delta_{13}}{B_{\mp}}\right) \sin \left(\frac{A L}{2}\right) \sin \left(\frac{B_{\mp} L}{2}\right) \\
& P_{4}= \pm J \sin \delta \sin \left(\frac{\Delta_{13} L}{2}\right)\left(\frac{\Delta_{12}}{A} \frac{\Delta_{13}}{B_{\mp}}\right) \sin \left(\frac{A L}{2}\right) \sin \left(\frac{B_{\mp} L}{2}\right)
\end{aligned}
$$

where $\Delta_{i j}=\Delta m_{i j}^{2} / 2 E, B_{ \pm}=\left|A \pm \Delta_{13}\right|$, and $A$ is the matter parameter defined by $A=\sqrt{2} G_{F} n_{e}$, with $G_{F}$ and $n_{e}$ the Fermi coupling constant, and number density of electrons in the earth.

We present a method to extract the unknown oscillation parameters $\theta_{13}$ and $\delta$ from the previous formula relying on Near and Far Detector data at a Neutrino Factory. This is an update of a previous study presented at NUFACT07 [2].

\section{Extraction of probability oscillation parameters}

The method relies on the extraction of the beam flux at the Near Detector (ND) and using these data to predict the number of events observed at a Far Detector (FD) as a function of energy. Monte Carlo simulations of Near and Far Detector at a Neutrino Factory were carried out, using realistic detector and flux assumptions at the default muon energy of $25 \mathrm{GeV}$ for a straight section of the decay ring at a Neutrino Factory of length $755 \mathrm{~m}$. A 50 kton MIND-like detector [3] like the one presented in the ISS detector report [4], with energy smearing of $\sigma(E) / E=0.55 / \sqrt{E}$, is used as a Far Detector at a distance of $4000 \mathrm{~km}$. The Near Detector is $100 \mathrm{~m}$ from the end of the straight section of the decay ring and is smeared with an energy resolution of $\sigma(E) / E=0.35 / \sqrt{E}$.

The smearing matrix obtained for the ND data was inverted and was used to extract an "unsmeared" ND signal. The estimated cross-sections were then unfolded from the data to obtain a ND flux estimation. This ND flux was propagated to the FD site to obtain the expected FD flux in the absence of oscillations. Inclusion of the cross-section data was then applied to obtain the expected number of FD events in the absence of oscillations for a perfect detector system. This is then multiplied by the expected oscillation probability for a number of scanned values of $\theta_{13}$ and $\delta$ and then the results are smeared according to the FD smearing matrix to take into account of detector effects. Comparison with the FD oscillated data is then used to obtain a global $\chi^{2}$. This $\chi^{2}$ is then minimised to obtain the most probable neutrino oscillation parameters that satisfy the data.

Figure 1 shows error contour plots for one example in which the oscillation parameters are extracted by this method (for $\theta_{13}=5^{\circ}$ and $\delta=45^{\circ}$ ) using the true Monte Carlo data from the ND that has not been smeared for detector effects (left) and smeared data using the expected smearing matrices and a simple inversion technique to unfold at the ND (right). A comparison of both methods allows one to compare the influence of the Near to Far Detector extrapolation in the 
extraction of the errors due to the oscillation fits. The influence of this extrapolation technique is seen to be very small, compared to the overall $\theta_{13}$ and $\delta$ errors.
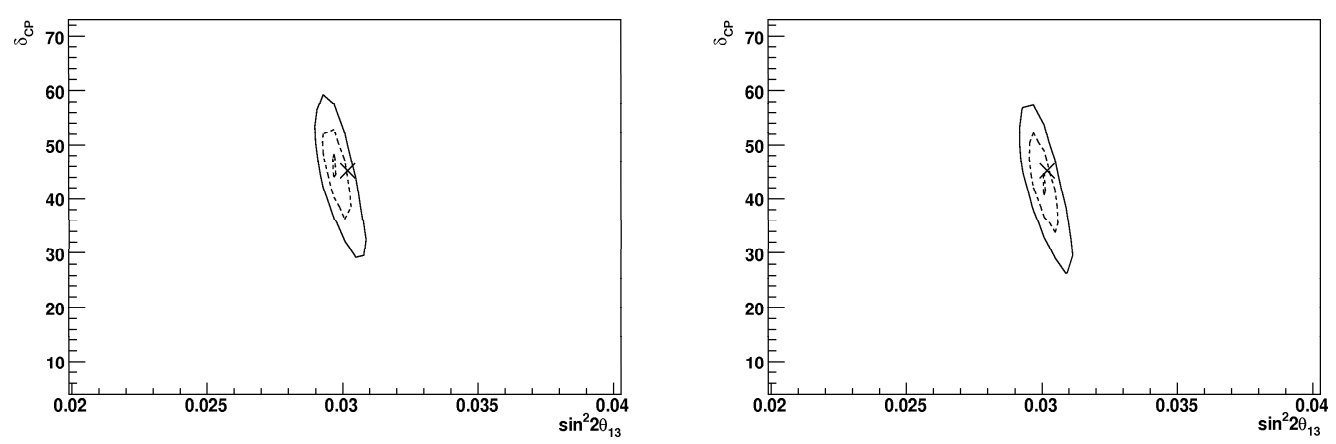

Figure 1: Contours of 1, 3, 5 sigma for the extraction of oscillation parameters from Near and Far Detector data without smearing for near detector effects (left) and with smearing (right). The input values of $\theta_{13}=$ $5^{\circ}$ and $\delta_{C P}=45^{\circ}$ are shown as a cross.

\section{Conclusions}

The effects of extrapolation of Near Detector data at a Neutrino Factory have been studied. An accurate extraction of the oscillation parameters from the fitted spectrum at a Far Detector has been achieved. The effect of realistic smearing of the energy on the detector interactions, in combination with an unfolding of the detector effects, is shown to have a negligible influence on the errors extracted from the oscillation fits.

\section{References}

[1] Minakata, H. and Nunokawa, H. (2001), JHEP 0110001.

[2] A. Laing, F.J.P. Soler, AIP Conf. Proc. 981, 166-168, (2008).

[3] A. Cervera-Villanueva, AIP Conf. Proc. 981, (2008), 178-180.

[4] T. Abe et al., ISS Detector Working Group, Detectors and flux instrumentation for future neutrino facilities, RAL-TR-2007-24, arXiv:0712.4129 [physics.ins-det] (2007). 